\title{
The Effect of Norepinephrine and Caffeic Acid on Cytokines and Nitric Oxide Production by Granulocytes is Age-Dependent
}

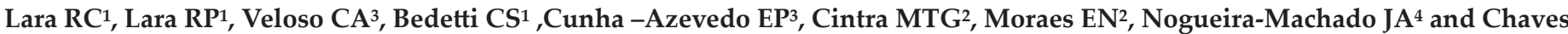 \\ $\mathrm{MM}^{1 *}$ \\ ${ }^{1}$ Laboratory of Aging and Correlated Diseases Biochemistry, Department of Biochemistry and Immunology, Institute of Biological Sciences, Federal \\ University of Minas Gerais, Brazil. \\ ${ }^{2}$ Reference center of the elderly Professor Caio Benjamin Dias and the Jenny de Andrade Faria Institute of Health Care for the Elderly, Hospital das \\ Clínicas, Federal University of Minas Gerais, Brazil. \\ ${ }^{3}$ University Center of Belo Horizonte, Av. Prof. Mario Werneck, Brazil. \\ ${ }^{4}$ Santa Casa Hospital of Belo Horizonte, Belo Horizonte, Brazil.
}

*Correspondence to: Míriam Martins Chaves, Department of Biochemistry and Immunology, Institute of Biological Sciences, Federal University of Minas Gerais, Postal Box, 486, 30161-970, Belo Horizonte, Minas Gerais, Brazil; Tel: 55313409 2660; Fax: 55313409 2614; E-mail: chavesmm@icb.ufmg.br

Received: December 22, 2017 Accepted: January 14, 2018; Published: January 23, 2018;

\begin{abstract}
There is a large increase in the number of elderly people in modern societies. This demographic phenomenon has been paralleled by an epidemic of chronic diseases and inflammatory process usually associated with advanced age. In this work we seek to fill a gap that still exists in the process of immunoregulation "old guy" looking to narrow the knowledge regarding their correlation with the immune system and the nervous system. The Nitric Oxide (NO) and Interleukins productions were studied in healthy subjects in age ranging from 20 to 80 years divided in three age groups: (20-39), (40-59) and (60-80) years. NO production was quantified in a Griess reaction. The results were expressed through the nitrite concentration calculated by linear regression using a standard curve obtained from a solution of sodium nitrite and 1mM RPMI. Interleukins 8, 10 and 4 (IL-8, IL-10 and IL-4) productions were measured by sandwich ELISA. Ours results demonstrated that Norepinephrine (NoAD) can no longer modulate the inflammatory cytokine profile and that there is a significant decay of the contribution of Caffeic Acid (CA) as an antioxidant and / or anti-inflammatory age-related. Thus, our results showed a lack of NoAD and CA modulation in human granulocyte in ageing dependent process.
\end{abstract}

Keywords: Aging, Granulocytes, Caffeic acid, Nitric oxide, Cytokines

\section{Significance Statement}

In view of the population aging, it is necessary to seek alternatives to improve the quality of life, as well as ways to ameliorate the diseases and pathologies associated with the increase of chronic inflammation and the drop in adaptive immunity inherent in the elderly. Thus, this work introduces the study on the modulating effect of caffeic acid and noradrenaline on the synthesis of nitric oxide and interleukins in human granular cells in different age groups. Following a widely accepted concept that peripheral cells may be a "window" within the central nervous system, correlating it simply to the immune system.

\section{Introduction}

Senescence is the natural ageing process, which progressively compromises physical and cognitive. According to World Health Organization (WHO), the elderly begins between 60 and 65 years. However, this is an old established for research purposes, since the aging process depends on three main factors classes: biological, psychological and social factors [1].

Ageing is a complex phenomenon that is still not completely understood. Age-related pathologies generally rise with approximately exponential kinetics beginning at approximately the mid-point of the species-specific life span (e.g., 50-60 years of age for humans) [2,3].

Progressive deterioration of innate and adaptive immune functions, a status termed 'immunosenescence', is associated with the higher frequency and severity of diseases in the elderly, such as chronic infections, cancer and autoimmune disorders. Age-related immunosenescence can be characterized by a decrease in adaptive immunity and increase in low-grade chronic inflammation, also referred to as 'inflammaging' [4]. Another significant aspect is the marked increase in oxidative stress generated during the aging process [5].

Studies by according to Chaves et al. demonstrated, in granulocytes, increased reactive oxygen species (ROS) after 40 years and nitrogen (RNS) from the age of 50. Concomitant with this increase, was observed a significant decrease in antioxidant power, suggesting an imbalance in cellular metabolic aging [6-8].

Currently, it is known that many stimuli from the central nervous system are capable of modulating an immune response. The hypothalamic-pituitary-adrenal (HPA)-is one of those responsible for several of the links between these two systems. This communication 
is bidirectional because cytokine activates neuronal axis and neurotransmitters may be produced by cells from immune system. Endorphins, thyrotropin, prostaglandins, growth hormone, and catecholamines are also part of this type of neuroimmunoendocrine response [9].

Norepinephrine (NoAD) is produced in response to physiological stress trigger and a broad spectrum of biochemical and physiological alterations including modulation of immunity [10]. It is believed that Caffeic acid (CA) able to prevent the neuroinflammationinduced acute, probably the long term neurodegenerative changes and attenuates lipopolysaccharide-induced sickness behaviour and neuroinflammation in mice [11].

It has also been suggested that cytokines have a pivotal role in ageing. Studies by according to Minciullo et al. [12] demonstrated that morbidity and mortality is associated with high level of IL-1, IL6,TNF- an IFN-Y in older people. The authors suggest that longevity depends on the balance between pro and anti-inflammatoy cytokines. Several therapeutic approaches have been suggested to modulate both inflammation and oxidative stress. Among them, caffeic acid (3,4-dihydroxycinnamic acid) is considered a compound with antiinflamatory and antioxidant properties. CA is believed to be able to prevent the neuroinflammation-induced acute and probably the long term neurodegenerative changes [13].

Catecholamines (CAs), including Norepinephrine (NoAD), is produced are the first hormones in response to physiological stress trigger and a broad spectrum of biochemical and physiological alterations including hyperglycemia and modulation of immunity [10] The Norepinephrine, major sympathetic neurotransmitters, modulates the immune system through interaction with adrenergic receptors $G$ protein-coupled ( $\alpha 1, \alpha 2, \beta 1, \beta 2, \beta 3)$. Thus, the occupation of these receptors stimulates or inhibits adenylate cyclase, which will influence the role of cytokines $[14,15]$.

Herein, we investigate in the present paper we study the effect of NoAD and CA in inducing the production of Nitric Oxide, IL-8, IL-4 and IL-10 in human granulocytes from different age range.

\section{Results}

\section{NoAD and CA lose the ability to modulate the production of NO during aging process}

Our results (Figure 1-panel A) showed that in the age group from 20-39 years showed a significant increase in the production of NO by granulocytes, when they were incubated with NoAD or CA, compared to the control group (RPMI + granulocytes) $(\mathrm{p}<0.05)$. The values expressed as millimolar (mM) were 11,51 for NoAD and 14,66 for CA in comparison with 5,87 for control group.

For group from 40-59 only CA was able to induce the increase in secretion of NO by granulocytes. (Figure 1-panel B). Both NoAD and $\mathrm{CA}$ were not able to activate the production of $\mathrm{NO}$ in granulocytes from age group of 60-80 years (Figure 1-panel C).
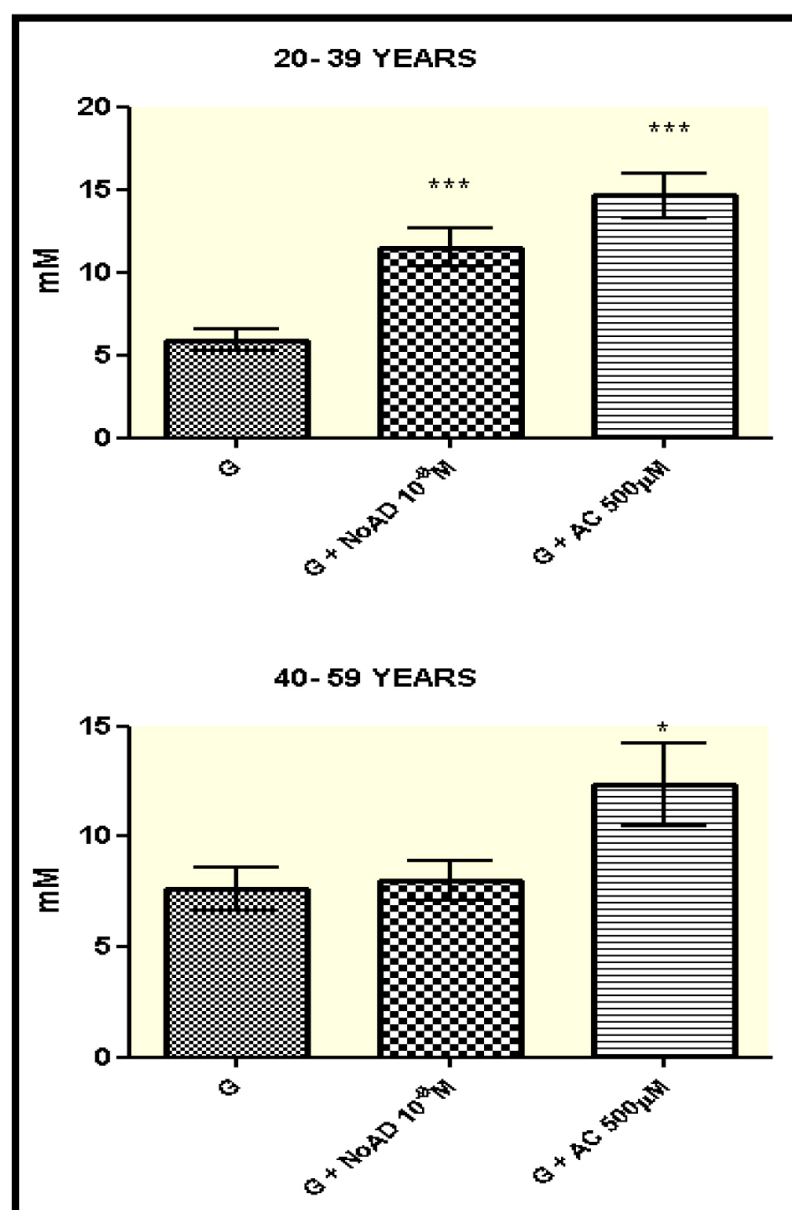

B

60- 80 YEARS

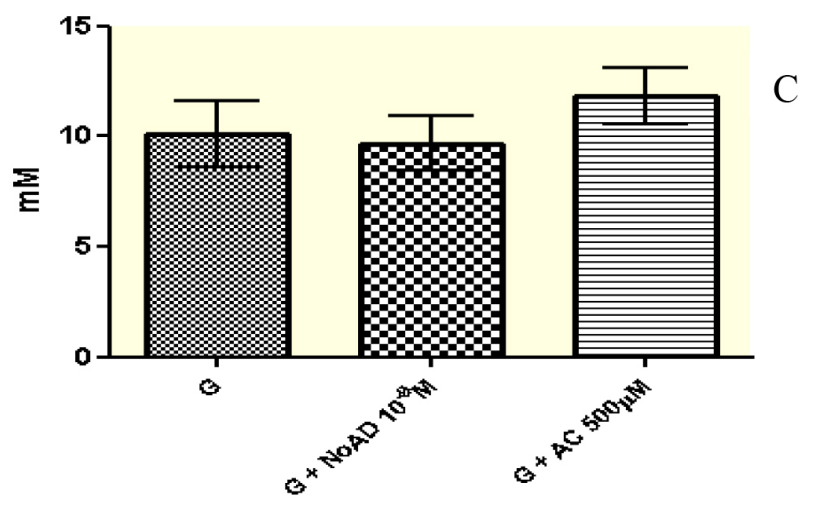

Figure 1. Norepinephrine and Caffeic Acid lose the ability to modulate the production of Nitric oxide during aging process. The values represent the mean \pm S.E. Nitric Oxide (NO) production was expressed as $\mathrm{mM} . \mathrm{G}=$ granulocyte, NoAD $=$ Norepinephrine, CA $=$ Caffeic Acid. ${ }^{*}$ Significant $(\mathrm{p}<0,05)$ when compared with control $(\mathrm{G})$ by ANOVA and Tukey post-test.

Effect of NoAD and CA on the production of IL-8: proinflammatory profile

Our results in the age group 20-39 years showed a significant difference $(\mathrm{p}<0.05)$ in IL8 production by granulocytes when they 
were stimulated by CA compared to the control group (RPMI + granulocytes) $(\mathrm{p}<0.05)$. The IL-8 production decreased $88 \%$. But NoAD activates the secretion of proinflammatory cytokine (IL-8) in $32 \%$ (Table 1, 2).

Table 1. Effect of Norepinephrine and Caffeic Acid on the production of IL-8 (proinflammatory profile) and IL-4 and IL-10 (anti-inflammatory profile)

\begin{tabular}{|c|c|c|c|}
\hline \multirow[t]{2}{*}{ Protocols } & \multicolumn{3}{|c|}{ 20-39 years } \\
\hline & IL-8 (pg/ml) & IL-4 (pg/ml) & IL-10 (pg/ml) \\
\hline G & $991 \pm 89$ & $172 \pm 19$ & $178 \pm 16$ \\
\hline $\mathrm{G}+$ NoAD & $1.313 \pm 120 *$ & $289 \pm 20 *$ & $169 \pm 10$ \\
\hline \multirow[t]{3}{*}{$\mathrm{G}+\mathrm{CA}$} & $120 \pm 95 * *$ & $163 \pm 17$ & $164 \pm 18$ \\
\hline & \multicolumn{3}{|c|}{$40-59$ years } \\
\hline & IL-8 (pg/ml) & IL-4 (pg/ml) & IL-10 (pg/ml) \\
\hline G & $193 \pm 14$ & $159 \pm 12$ & $117 \pm 14$ \\
\hline $\mathrm{G}+$ NoAD & $246 \pm 17 *$ & $165 \pm 14$ & $107 \pm 9$ \\
\hline \multirow[t]{3}{*}{$\mathrm{G}+\mathrm{CA}$} & $132 \pm 11^{*}$ & $153 \pm 16$ & $65 \pm 4 *$ \\
\hline & \multicolumn{3}{|c|}{$60-80$ years } \\
\hline & IL-8 (pg/ml) & IL-4 (pg/ml) & IL-10 (pg/ml) \\
\hline G & $75 \pm 3$ & $113 \pm 10$ & $94 \pm 5$ \\
\hline $\mathrm{G}+\mathrm{NoAD}$ & $75 \pm 4$ & $130 \pm 12$ & $84 \pm 4$ \\
\hline $\mathrm{G}+\mathrm{CA}$ & $58 \pm 2 *$ & $104 \pm 5$ & $64 \pm 3 *$ \\
\hline
\end{tabular}

The values represent the mean \pm S.E. Interleukin production was expressed as $\mathrm{pg} / \mathrm{ml}$. G $=$ granulocyte, NoAD $=$ Norepinephrine, $\mathrm{Ac}=$ Caffeic Acid. $\left({ }^{*}\right)$ and $\left({ }^{* *}\right)$ was Significant $(p<0.05)$ and $(p<0.01)$ respectively, when compared with control $(G)$ by ANOVA and Tukey post-test

Table 2. Demographic characteristics of the studied groups.

\begin{tabular}{|c|c|c|c|}
\hline Subjects & 30 Male & & 30 Female \\
\hline Parameters & Values (mean \pm S.D.) & & Values (mean \pm S.D.) \\
\hline \multicolumn{4}{|c|}{ Age groups (years) } \\
\hline $20-39$ & $32.3 \pm 6$ & ns & $29.8 \pm 5$ \\
\hline $40-59$ & $52.1 \pm 4$ & ns & $50.2 \pm 5$ \\
\hline $60-80$ & $76,3 \pm 7$ & ns & $66.9 \pm 6$ \\
\hline MEDIUM & $53,6 \pm 6$ & ns & $48.9 \pm 5$ \\
\hline \multicolumn{4}{|c|}{ Body Mass Index $\left(\mathrm{kg} / \mathrm{m}^{2}\right)$} \\
\hline $20-39$ & $22.4 \pm 2$ & ns & $21.8 \pm 3$ \\
\hline $40-59$ & $28.8 \pm 3$ & ns & $27.5 \pm 3$ \\
\hline $60-80$ & $24.4 \pm 3$ & ns & $28.5 \pm 4$ \\
\hline MEDIUM & $25.2 \pm 3$ & ns & $25.9 \pm 3$ \\
\hline \multicolumn{4}{|c|}{ Glycohemoglobin (GHb\%) } \\
\hline $20-39$ & $4.0 \pm 0,1$ & ns & $3.9 \pm 0,3$ \\
\hline $40-59$ & $5.4 \pm 0,3$ & ns & $5.5 \pm 0,3$ \\
\hline $60-80$ & $5.5 \pm 0,4$ & ns & $5.6 \pm 0,2$ \\
\hline MEDIUM & $4.9 \pm 0,3$ & ns & $5.0 \pm 0,3$ \\
\hline \multicolumn{4}{|c|}{ Triglycerides (mg/dL) } \\
\hline $20-39$ & $102.4 \pm 38$ & ns & $101.8 \pm 29$ \\
\hline $40-59$ & $130.0 \pm 57$ & ns & $127.7 \pm 43$ \\
\hline $60-80$ & $164.3 \pm 20$ & ns & $153.5 \pm 23$ \\
\hline MEDIUM & $132.2 \pm 38$ & ns & $127.7 \pm 32$ \\
\hline
\end{tabular}

\begin{tabular}{|c|c|c|c|}
\hline Subjects & 30 Male & & 30 Female \\
\hline \multicolumn{4}{|c|}{ Serum creatinine $(\mathrm{mg} / \mathrm{dL})$} \\
\hline $20-39$ & $0.979 \pm 0,161$ & $\mathrm{~ns}$ & $0.821 \pm 0,161$ \\
\hline $40-59$ & $0.921 \pm 0,152$ & $\mathrm{~ns}$ & $0.800 \pm 0,152$ \\
\hline $60-80$ & $0.994 \pm 0,176$ & $\mathrm{~ns}$ & $0.900 \pm 0,170$ \\
\hline MEDIUM & $0.965 \pm 0,163$ & $\mathrm{~ns}$ & $0.840 \pm 0,161$ \\
\hline
\end{tabular}

ns= non-significant by the Mann-Whitney test.

Body Mass index: Normal range $=18,5-24,99 \mathrm{~kg} / \mathrm{m}^{2}$ (World Health Organization, 2000)

Glycohemoglobin: Normal value $\leq 5,7 \%$ (American Diabetes Association, 2011)

Triglycerides: Normal range $<150 \mathrm{mg} / \mathrm{dL}$ (American Heart Association, 2009)

Serum creatinine: Normal range: $0,5-1,5 \mathrm{mg} / \mathrm{dL}$ for males and $0,6-1,2 \mathrm{mg} / \mathrm{dL}$ for females (NCCLS, 2000)

Our results in the age group $40-59$ years showed difference $(\mathrm{p}<0.05)$ in the production of IL- 8 by granulocytes when they were stimulated by NoAD (increase of $27 \%$ ) or CA decrease of $31 \%$ ) (Table 1). In the age group of $60-80$ years found no significant difference ( $p>0.05$ ) was observed for the production of IL- 8 when granulocytes were incubated with NoAD. But, CA inhibited IL-8 secretion $22 \%$ $(\mathrm{p}<0.05)$ in relation the control group.

\section{Effect of NoAD and CA on the production of IL-4 and IL-10: anti-inflammatory profiles}

Our results in the age group from 20-39 years, NoAD induced an IL-4's secretion (68\%) by granulocytes in relation to the control group (RPMI + granulocytes) $(\mathrm{p}<0.05)$. CA was not effective on IL-4's secretion. The secretion of IL-10 by granulocytes showed no significant difference $(\mathrm{p}>0.05)$ when that cells were stimulated by NoAD or CA (Table 1).

Our results showed age group from 40 to 80 years showed that IL- 4 production by granulocytes stimulated by in the presence of NoAD or CA was not affected ( $p>0.05$ ) (Table 1). Similar results were observed the production of for IL-10 was not significant $(\mathrm{p}>0.05)$ granulocytes were incubated with NoAD (Table 2). In the contrast, we observed a significant decrease in IL-10's secretion by granulocytes $(\mathrm{p}<0.05)$ incubated with CA ( $44 \%$ and $32 \%$ ) in the age group from $40-59$ and 60-80 years respectively.

\section{Inhibitory effect of CA on the action of NoAD: process aging dependent?}

Our findings clearly show a decrease of CA action on stimulatory effect of NoAD on NO production by human granulocytes during the aging process. We observed in the group from $20-39$ years $(49 \%$ activation), $40-59$ years (34\% inhibition) and 60-80 years (38\% inhibition) effect of CA on NoAD in human granulocytes (Figure 2).

When evaluating the profile of interleukin pro and antiinflammatory found that a decrease in the running action of CA on the stimulatory action of NoAD in the production of IL- 8 by human granulocytes during aging 91\% (20-39 years) 54\% (40-59 years) and $16 \%$ (60-80 years) (Figure 3 and panel A). Evaluating the antiinflammatory interleukins also found the same profile. IL-4 ranges of $20-39$ years (22\%), $40-59$ years (17\%) and 60-80 years (3\%) inhibition 
(Figure 3 and panel B). Our findings with IL-10 showed range of 2039 years (16\% activation), $40-59$ years (18\% inhibition) and $60-80$ years (16\% inhibition) effect of CA on NoAD in human granulocytes. (Figure 3 and panel C).

\section{Modulation of the AC on NOAD in the process of production of NO}

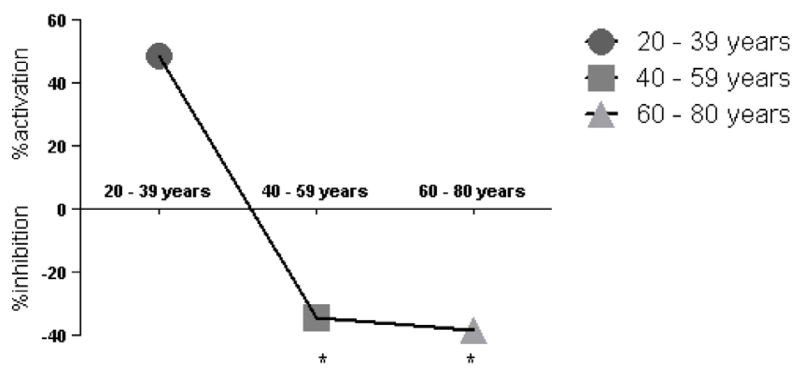

Figure 2. Lack modulating effect of Caffeic Acid on the action of Norepinephrine in Nitric Oxide production: process aging dependent. The values represent the mean \pm S.E. Nitric Oxide (NO) production was expressed as $\mathrm{mM}^{*}$ Significant $(\mathrm{p}<0,05)$ when compared with control $(\mathrm{G})$ by ANOVA and Tukey post-test.

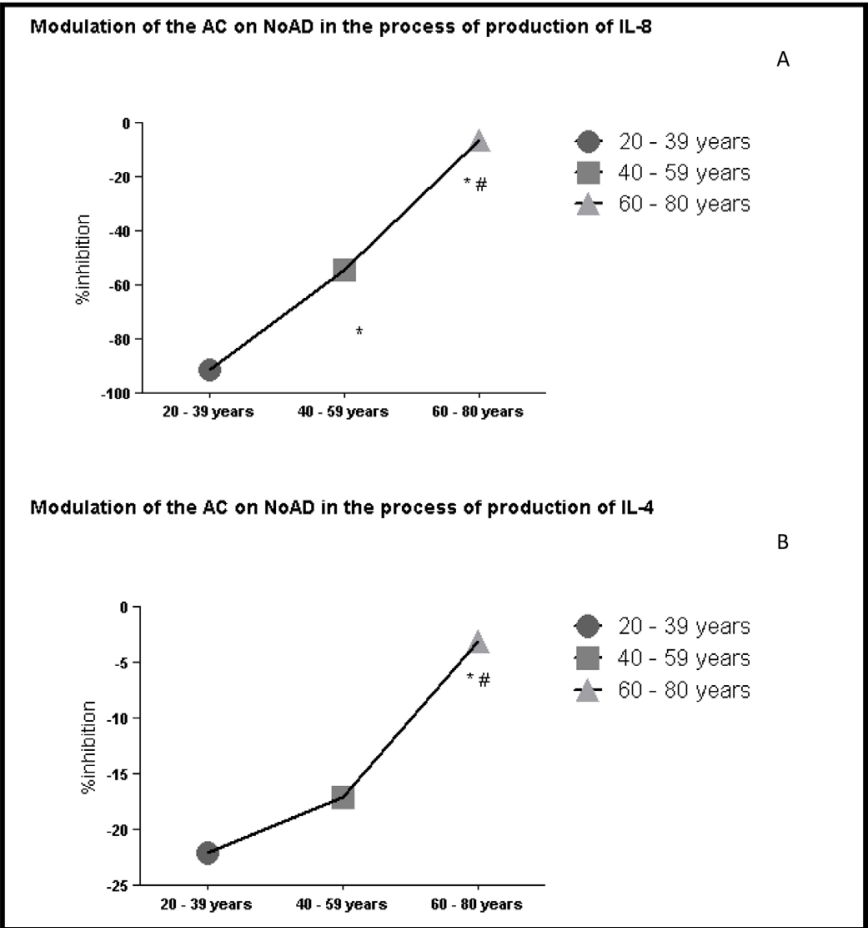

Figure 3. Lack modulating effect of Caffeic Acid on the action of Norepinephrine in Interleukins production: process aging dependent. *Significant $(p<0,05)$ when compared with control $(\mathrm{G})$ by ANOVA and Tukey post-test.

\section{Discussion}

The immune system undergoes profound transformations with age, and response patterns to immunological challenges are therefore highly age dependent. Changes that occur in humans after the age of 50 years have received particular attention because of their clinical impact. Such changes have been globally called 'immunosenescence. The most widely appreciated consequence of advanced age is diminished effectiveness of the immune system. However, immunosenescence is multifaceted and also includes an enhanced susceptibility to autoimmunity that is conceptually difficult to reconcile with the impaired responsiveness of the adaptive immune system as well as constitutive low-grade inflammation that may contribute to a plethora of degenerative diseases, including cardiovascular disease, neurodegenerative syndromes and age-specific ailments such as frailty [16].

In this context, the immune system has many properties of great importance for survival. The various stimuli captured by the brain during different situations can act beneficial or detrimental on the immune system, triggering a series of reactions with important consequences. Among these stimuli stress deserves [17].

NO is a key signaling molecule in the cardiovascular, immune and central nervous systems (CNS), and crucial steps in the regulation of NO bioavailability in health and disease are well characterized [18]. The generation of NO by neutrophils is involved in its antimicrobial function. This molecule has been identified as a modulator of various signaling cascades that regulate various functions such as adhesion, chemotaxis, phagocytosis, and respiratory burst, modulation of apoptosis and generation of free radicals [19]. Furthermore, NO has a complex physiological role in the CNS and regulating neuroendocrine functions. It has a substantial capacity to affect dopaminergic, serotonergic and noradrenergic neurotransmission [20]. Studies demonstrated that CA modulates endothelial NO production in a dose-dependent manner. In experimental model, kidney ischemia-reperfusion injury, CA in which this compound significantly reduced tubular cell apoptosis and granulocyte infiltration [21]. Our results (Figure 1-panel A) demonstrate that CA is able to activates NO releasing from granulocytes in 20-39 age group.

We also found that there was a significant increase $(\mathrm{p}<0.05)$ in the production of NO in the age group from 20-39 years when granulocytes were incubated with NoAD (Figure 1-panel A). GadekMichalska and Bugagiski have reported that NoAD increases the in vitro synthesis of $\mathrm{NO}$ in the medial basal hypothalamus through a 1 -adrenergic receptors [20].

CA increased NO releasing by granulocytes in 20-39 and 40-59 ages groups in similar manner (Figure 1-panel B). CA was able to increase NO levels, thus demonstrating its continuing role as a cell protector, mainly neuroprotective in this age group.

However, when we analyze the in the presence of effect of NoAD verified that this was not significantly $(p>0.05)$, the production of NO was not significantly found in younger individuals (Figure 2-Panels A and B). Here we try to identify some things that may be guiding this failure: 1 - failure of the regulatory mechanisms of the HPA axis, 2- a possible inefficiency in awareness receptor a 1-adrenergic stimulation, 3- a deficiency in the NoAD on NO synthase (the enzyme responsible for the synthesis NO). In the Age group from 60-80 years showed that NoAD and CA were not able to change $(p>0.05)$ the production of NO (Figure 1-panel $\mathrm{C})$. These results may signal that during the aging process there is a reduction of the sympathetic response that may be justified mainly by the decrease in sensitization of adrenergic receptors a 1- adrenergic. It 
is suggested that changes in the production of NO leads to activation of iNOS (induced NO synthase) generating a pro-inflammatory response that may be one of the factors that contribute to aging and neurodegenerative diseases [22].

Neuroendocrine hormones are considered to play major roles in the regulation of homeostasis under stressful environments. Catecholamines (CAs) including NoAD are the first hormones in response to physiological stress trigger a broad spectrum of biochemical and physiological alterations including hyperglycemia and modulation of immunity. Researchers by Zhang, et al. demonstrated the inhibited immune responses and resistance against bacterial infection were observed in both Litopenaeus vannamei and $M$. rosenbergii received NoAD by injection [11]. These results illustrate the importance of the sympathetic nervous system in modulating immunological pathological and non-pathological conditions.

Our results showed aged group from 20-39 years increased $(\mathrm{p}<0.05)$ the production of IL-8 (proinflammatory cytokine) when granulocytes were stimulated with NoAD (Table 1). When evaluating the production of anti-inflammatory cytokines we observed an increased $(\mathrm{p}<0.05)$ the production of IL-4 but not IL-10 (Table 1). These results show that at this age the IL- 4 is who participates in these anti-inflammatory mechanisms and that really CAs may modulate the inflammatory profile in young individuals which realize a balance between cytokines pro and anti-inflammatory. These results demonstrate possibly efficient communication between the immune system and the regulation of the HPA axis by neurohormones such as NoAD to maintain balance pro and anti-inflammatory.

Oxidative stress can increase cytokine production via several different mechanisms. Therefore, during the inflammatory process, oxidizing molecules increase the production of interleukins (IL-8 and IL-6) and TNF-alpha in response to inflammatory stimuli and cytokines increase the production of oxidants setting up a vicious cycle $[23,24]$. In addition to antioxidant defense acts directly or indirectly protecting the body from attacks of cytokines and oxidizing. It is believed that the antioxidants protect indirectly by reducing the activation of signaling pathways, preventing therefore the stimulation of cytokine oxidants [25].

Our results showed that CA significantly decreased $(\mathrm{p}<0.05)$, the production of IL-8 in the age group of 20-39 years (Table 1). However we did not observe significant results $(\mathrm{p}>0.05)$, when evaluating the production of IL-4 and IL-10. These results showed that CA can act as a potent anti-inflammatory phenolic acid as this could reduce by more than $90 \%$ the production of IL-8 in young individuals (Table 1).

Assessing the age group of 40-59 years found that NoAD increased $(p<0.05)$, the production of IL- 8 but did not change $(p>0.05)$ the production of both IL-4 and IL-10 (Table 1). The results for CA have shown that this did not affect the production of IL-4, but decreases the production of IL-8 and IL-10 (Table 1).

Aged 60-80 years found that NoAD did not change $(p>0.05)$ the production of IL- 8 and neither of the cytokines ( $p>0.05$ ), IL- 4 and IL10 (Table 1). The results for CA have shown that this did not affect the production of IL- 4 but decreases the production of IL- 8 and IL-10, as seen in the age group 40-59 years (Table 1).

Our results are reinforce previous results Chaves et. al. on deficiency of IL-10 during the aging process. Moreover, this reduced production of IL- 8 by CA may be related to antioxidant mechanisms that are deficient in aging and which is a potent anti-oxidant contribute to the reduction of the framework [6,7].Thus, our results showed here a profile change where we find a framework favoring the balance pro-inflammatory and oxidative signs of senescence, which is characterized as a chronic inflammatory process and stress.

Aging results in a progressive decrease and general functions of the body, decreasing the ability to react to adaptive changes, and maintain homeostasis. Evidences show that the immune system can initiate state oxidative and inflammatory uncontrolled factors which are linked to the aging process and affect all cells of the body but especially neuroimmunoendocrine system cells [26]. Another aspect is that the antioxidant systems may exert a neuroprotective role by protecting the nervous tissue degeneration caused by ischemia or as a result of chronic neurodegenerative diseases [27]. Endogenous antioxidant defenses can be both non-enzymatic (uric acid, glutathione, bilirubin, thiols, albumin, vitamins and phenols) and enzymatic (superoxide dismutases, glutathione peroxidase and catalase) [28]. The levels of glutathione and these antioxidant enzymes are much lower in the CNS as compared to erythrocytes and peripheral tissues $[29,30]$.

Next context to evaluate the modulating effect of CA on the action of NoAD in the production of NO found that aged group from 20-39 years there is an upregulation (increased production of nitric oxide) which can configure the protective action of $\mathrm{NO}$ in this age group (Figure 2). However, when we analyzed the age groups from 40-80 years found an inhibition $(\mathrm{p}<0.05)$ NO production when CA was incubated with NoAD compared with the age group from 20-39 years (Figure 2). This table denotes a loss of antioxidant CA in these age groups which can cause systemic and nervous damage.

In evaluating the modulating action the $\mathrm{CA}$ on NoAD aged group from 20-39 years found that it is able to increase the production of cytokines both pro and anti-inflammatory setting a balance between these systems (Figure 3 - panels A, B and C). When we evaluated the age group from 40-59 years observed a significant decrease $(\mathrm{p}<0.05)$ the production of IL- 8 and IL-10 and one did not change ( $p>0.05)$ the production of IL-4 when compared to age group from 20-39 years (Figure 3-panels A, B and C). In the age group 60-80 years observed a decrease $(\mathrm{p}<0.05)$ marked production of both interleukins pro (IL8) as anti-inflammatory (IL-4 and IL-10) (Figure 3-panels A, B and C). In establishing this framework we realized that NoAD can no longer modulate the inflammatory cytokine profile and that there is a significant decay of the contribution of CA as an antioxidant and / or anti-inflammatory.

Therefore, our findings aid in the elucidation of important metabolic points that are part of neuroimmunoendocrine regulation. The aging reflects the sum of many changes that occur in humans during life. The association between age and decreased function of the immune system increases susceptibility to several neurodegenerative diseases. Within this context our work raised several questions in 
which demonstrated that there is need for further study the molecular mechanisms that are involved in neuroimmunoendocrine systems especially with regard to the adrenergic pathway because it proved to be important in maintaining homeostasis between the immune endocrine and nervous systems.

\section{Experimental Procedure}

\section{Reagents}

The concentrations of Interleukin 8 (IL-8), Interleukin 4 (IL-4), and Interleukin 10 (IL-10) were measured by sandwich ELISA using kit supplied by Assay Designs (Ann Arbor, MI, USA).

\section{Subjects}

The Ethical Committees of Federal University of Minas Gerais (UFMG) approved this study (CAAE 0663.0.203.000-11). A detailed medical history, physical examination and laboratory data for each subject were recorded four weeks before entering the study. Appropriate informed consent was obtained from each participant. According to the Senior protocol for the study of immune function in the elderly, [31], 45 people were excluded from a total of 105 because they showed conditions which were not suitable for the admission criteria (i.e. smokers, infections, inflammation, malignancy, lymphoproliferative disorders, arteriosclerosis, cardiac insufficiency, hypertension, dementia, pregnancy, alcoholism and drug abuse) and/or were taking known drugs that directly influence the immune function. Subjects were divided into three age groups with 10 males and 10 females in each, aiming equality in the number of subjects from each group, and selected by authors ENM and MTGC (Reference Center of Age - Professor Caio Benjamin Dias from Hospital das Clínicas-UFMG). These subjects live in Belo Horizonte, Brazil. The demographic characteristics of the population are shown in Table 2.

\section{Cell separation}

The granulocytes were purified from $10 \mathrm{ml}$ of heparinized venous blood from subjects distributed into tree age groups: 20-39 years, 4059 years and $60-80$ years. The cells were isolated by Ficoll-Hypaque gradient according to Bicalho et al. [32] with slight modifications. Briefly, the neutrophils were separated through the centrifugation of cells over two gradients with density of 1.08 and 1.13 in order to obtain, simultaneously, the separation of mononuclear cells and granulocytes, respectively. The purity of the preparation of granulocytes was 95$100 \%$. The viability of each sample was always greater than $98 \%$ as determined by the Trypan Blue exclusion test.

\section{Dose-response curve}

A dose-response curve was obtained by adding increasing concentrations of $\operatorname{NoAD}\left(10^{-12} \mathrm{M}, 10^{-11} \mathrm{M}, 10^{-10} \mathrm{M}, 10^{-9} \mathrm{M}, 10^{-8} \mathrm{M}, 10^{-7}\right.$ $\mathrm{M})$ and $\mathrm{CA}(13 \mu \mathrm{M}, 32 \mu \mathrm{M}, 80 \mu \mathrm{M}, 200 \mu \mathrm{M}, 500 \mu \mathrm{M}, 1250 \mu \mathrm{M})$. The best concentration of NoAD was $\left(10^{-9} \mathrm{M}\right)$ and $\mathrm{CA}(500 \mu \mathrm{M})$, which reflected a lower cell death ( $5 \%$ and $7 \%$ respectively) and a greater biological effect.

\section{Nitric Oxide (NO) production-Griess reaction}

The quantification of nitric oxide was carried out by measuring nitrite second studies by Griess [33]. $100 \mu \mathrm{L}$ granulocytes were incubated in the presence or absence of NoAD $\left(10^{-9} \mathrm{M}\right)$ and CA $(500 \mu \mathrm{M})$. In all experiments the final volume was adjusted to $300 \mu \mathrm{l}$ with RPMI pH 7.4. Granulocytes were maintained in cell culture plates of 24 wells, incubated at $37^{\circ} \mathrm{C}$ and $5 \% \mathrm{CO}_{2}$ for $16 \mathrm{~h}$. After these 16 hours, the granulocytes were centrifuged at $2500 \mathrm{rpm}$ for 15 minutes. The supernatant was collected and used for determination of nitrite. The pellet was resuspended in $200 \mu$ l of RPMI pH 7.4 and immediately subjected to cell viability analysis. For measurement of nitrite, $100 \mu \mathrm{L}$ of supernatant was used, which were placed in 96-well plates. To the supernatant was added $100 \mu \mathrm{L}$ of a solution Griess, which is formed sulfanilamide $1 \%$ in $2.5 \%$ phosphoric acid and $0.1 \%$ naphthylethylenediamine in $2.5 \%$ phosphoric acid in a $1: 1$ ratio. The contents of the plate were analyzed by ELISA reader at $540 \mathrm{~nm}$. The nitrite concentration was calculated by linear regression using a standard curve obtained from a solution of sodium nitrite and $1 \mathrm{mM}$ RPMI.

\section{Determination of IL-8, IL-4 and IL-10 in the supernatant of Granulocytes}

Aliquots $(3 \mathrm{~mL})$ of the suspension of granulocytes $\left(1 \mathrm{x} 10^{6}\right.$ cells/100 $\mu \mathrm{L}$ RPMI-1640 medium) were incubated in the presence or absence of NoAD or CA under $5 \% \mathrm{CO}_{2}$ for 16 hours at $37^{\circ} \mathrm{C}$. Following incubation, the cells were centrifuged and the supernatant, collected. The concentrations of IL-8, IL-4 and IL-10 were measured by sandwich ELISA using kit supplied by Assay Designs (Ann Arbor, MI, USA) according to supplier instructions. The levels of cytokine were determined using standard curves for IL-8, IL-4 and IL-10, respectively.

\section{Statistical analysis}

All results were analyzed by ANOVA and Tukey post-test using GraphPad Prism version 5.00 for windows (San Diego, CA). $\mathrm{p}<0.05$ was considered to indicate statistical significance.

\section{Conflict of interest}

The authors confirm that this article content has no conflict of interest.

\section{Acknowledgements}

The authors thank Gláucia A.A. Carvalho for excellent technical assistance. FAPEMIG CNPq and IVC Health- testes em saúde humana LTDA supported this paper.

\section{References}

1. Pereira A, Freitas C, Mendonça C, Marçal F, Souza J, et al. (2004) Aging, stress and society: a psychoneuroendocrine view. Science and Cognition 1: 34-53.

2. Vitale G, Salviole S, Franceschi C (2013) Oxidative stress and the ageing endocrine system, Nature Rev Endocrinol 9: 228-240. [crossref]

3. Campisi J (2013) Aging, Cellular Senescence, and Cancer. Annu Rev Physiol 75: 685-705. [crossref]

4. Hoffmann J, Spyridopoulos I (2015) Senescent cytotoxic T cells in acute myocardial infarction: innocent bystanders or the horsemen of apocalypse? Cell Mol Immunol 12: 510-512. [crossref]

5. Harman D (1981) The aging process. Medical Science Proc Natl Sci US 78: 7124 7128. [crossref]

6. Chaves MM, Costa DC, Oliveira BF, Rocha MI, Nogueira-Machado JA (2009) Role PKA and p38 MAPK on ROS production in neutrophil age-related: Lack of IL-10 effect in older subjects. Mech Ageing Dev 130: 588-591. [crossref] 
7. Chaves MM, Rocha-Vieira E, Lima e Silva R, Machado-Nogueira JA (1998) Host defenses in aged: evaluation of the balance between oxidizing species generation and reducing power in phagocyting human granulocytes. Mech Ageing Dev 104: $103-109$

8. Chaves MM, Rocha-Vieira E, Reis AP, Lima e Silva R, Gerzstein NC, et al. (2000) Increase of reative oxygen (ROS) and nitrogen (RNS) species generated by phagocyting granulocytes related to aged. Mech Ageing Dev 119: 1-18.

9. Alves GJ, Palermo J (2007) Neuroimmunomodulation: the cross-talk between the nervous and immune system. Rev Brasil Psiquiatr 29.

10. Chang CC, Tsai WL, Jiang JR, Cheng W (2015) The acute modulation of norepinephrine on immune responses and genes expressions via adrenergic receptors in the giant freshwater prawn, Macrobrachium rosenbergii. Fish Shellfish Immunol 46: 459-467. [crossref]

11. Zhang M, Zhou J, Wang L, Li B, Guo J, et al. (2014) Caffeic Acid Reduces Cutaneous Tumor Necrosis Factor Alpha (TNF $\alpha$ ),IL-6 and IL-1 $\beta$ Levels and Ameliorates Skin Edema in Acute and Chronic Model of Cutaneous Inflammation in Mice. Biol Pharm Bull 37: 347-354. [crossref]

12. Minciullo PL, Catalano A, Mandraffino G, Casciaro M, Crucitti A, et al. (2015) Inflammaging and Anti-Inflammaging: The Role of Cytokines in Extreme Longevity. Arch Immunol Ther Exp (Warsz) 64: 111-126. [crossref]

13. Mallik SB, Mudgal J, Nampoothiri M, Hall S, Dukie SA, et al. (2016) Caffeic acid attenuates lipopolysaccharide-induced sickness behaviour and neuroinflammation in mice. Neurosci Lett 632: 218-223. [crossref]

14. Tracey KJ (2009) Reflex control of immunity. Nat Rev Immunol 9: 418-428. [crossref]

15. Szelényi J, Vizi ES (2007) The catecholamine cytokine balance: interaction between the brain and the immune system. Ann N Y Acad Sci 1113: 311-324. [crossref]

16. Goronzy JJ, Weyand CM (2013) Understanding immunosenescence to improve responses to vaccines. Nat Immunol 14: 428-436. [crossref]

17. Bauer ME (2005) Stress, glucocorticoids and ageing of the immune system stress. Stress 8: 69-83. [crossref]

18. Lundberg JO, Gladwin MT, Weitzberg E (2015) Strategies to increase nitric oxide signalling in cardiovascular disease. Nat Rev Drug Discov 14: 623-641. [crossref]

19. Kobayashi SD, Deleo FR (2009) Role of neutrophils in innate immunity: a systems biology-level approach. Wiley Interdiscip Rev Syst Biol Med 1: 309-331. [crossref]

20. Gadek-Michalska KA, Bugajski G (2008) Nitric oxide in the adrenergic-and crhinduced activation of hypothalamic-pituitary-adrenal axis. J Physiol Pharmacol 59: 365-378. [crossref]
21. Migliori M, Cantaluppi V, Mannari C, Bertelli AA, Medica D, et al. (2015) Caffeic Acid, a Phenol Found in White Wine, modulates Endothelial Nitric Oxide Production and Protects from Oxidative Stress-Associated. Endothelial Cell Injury. PLoS One 10. [crossref]

22. Navarro A, Boveris A (2008) Mitochondrial nitric oxide synthase mitochondrial brain dysfunction in aging and mitochondrial-targed antioxidants. Adv Drug Deliv Rev 60: 1534-1544. [crossref]

23. Elmarakby AA, Sullivan JC (2012) Relationship between Oxidative Stress and Inflammatory Cytokines in Diabetic Nephropathy. Cardiovasc Ther 30: 49-59. [crossref]

24. Bianca VD, Dusi S, Bianchini E (1999) Beta- amyloide activates the o-2 forming NADPHoxidase in microglia, monocytes,neutrophils. A possible inflammatory mechanism of neuronal damage in Alzheimer's disease. J Biol Chem 274: 1549315499. [crossref]

25. Grimble RF (1994) Nutritional antioxidants and the modulation of inflammation: theory and practice. New Horiz 2: 175-185. [crossref]

26. De La Fuente M, Miquel J (2009) An update the oxidation-inflammation theory of aging: the involvement of the immune system in oxi-inflamm-aging. Curr Pharm Des 15: 3003-3026. [crossref]

27. Rafii MS, Aisen PS (2009) Recent developments in Alzheimer's disease therapeutics. BMC Med 7: 7. [crossref]

28. Fusco D, Colloca G, Lo Monaco MR, Cesar M (2007) Effects of antioxidant supplementation on the aging process, Clin Interv Aging 2: 377-387. [crossref]

29. Floyd RA , Hensley K (2002) Oxidative stress in brain aging. Implications for therapeutics of neurodegenerative diseases. Neurobiol Aging 23: 795-807. [crossref]

30. Chalimoniuk M, Jagsz S, Sadowska-Krepa E, Chrapusta SJ, Klapcinska B, et al. (2015) Diversity of endurance training effects on antioxidant defenses and Oxidative damage in different brain regions of adolescent male rats. $J$ Physiol Pharmacol 66: 539-547. [crossref]

31. Ligthrt GJ, Corberand JX, Fournier C, Galanaud P, Hijmans IN, et al. (1984) Admission criteria for immunogerontological studies in man the senieur protocol. Mech Ageing Dev 28: 47-55. [crossref]

32. Bicalho HMS, Gontijo MC, Nogueira-Machado JA (1981) A simple techinique for simultaneous human leockocytes separation. J Immunol 40: 115-116.

33. Griess P (1864) One a new of bodies in which nitrogen is substituted for hydrogen. Philos Trans R Soc Lond 154: 667-723.

\section{Citation:}

Lara RC, Lara RP, Veloso CA, Bedetti CS ,Cunha - Azevedo EP, Cintra MTG ,Moraes EN, Nogueira-Machado JA and Chaves MM (2018) The Effect of Norepinephrine and Caffeic Acid on Cytokines and Nitric Oxide Production by Granulocytes is Age-Dependent. Ageing Sci Ment Health Stud Volume 2(1): 1-7 